\title{
The Necessary Observation Space for the Lowest GDOP in 2-D Wireless Location Systems
}

\author{
Hong-Tao He \\ Beijing University of Posts and Telemommunications, \\ Xitucheng Road NO.10, Haidian District \\ Beijing city, China \\ bupt_hongtaohe@163.com \\ Qing-Yi Quan \\ Beijing University of Posts and Telemommunications, \\ Xitucheng Road NO.10, Haidian District \\ Beijing city, China \\ qyquan@bupt.edu.cn
}

\author{
Wen-Bo Wang \\ Beijing University of Posts and Telemommunications, \\ Xitucheng Road NO.10, Haidian District \\ Beijing city, China \\ wbwang@bupt.edu.cn
}

\begin{abstract}
The necessary observation space to achieve the lowest geometric dilution of precision (GDOP) based absolute-range measurement in two-dimension (2-D) wireless location systems is researched. Angle of coverage (AOC) is introduced as a parameter to describe the observation space. In fact, AOC reflects the geometric relationship between the target and the measuring points. A new clever geometric relationship between a target and $N$ measuring points is proposed in this paper. And then, this geometric relationship is proved to have the minimum AOC to achieve the lowest GDOP in 2-D wireless location systems. It is shown that when the AOC is less than the minimum AOC, the lowest GDOP of $2 / \sqrt{N}$ could not be obtained. So the minimum AOC is called the necessary AOC. The necessary AOC is equal to $\pi / 2$ when $\mathrm{N}$ is even, while the necessary $\mathrm{AOC}$ is monotonically decreasing to $\pi / 2$ when $\mathrm{N}$ is odd. When the AOC is less than the necessary AOC, the optimal geometry is derived as well as its new low bound of GDOP.
\end{abstract}

Keywords-Angle of coverage; the lowest GDOP; 2-D location system; absolute-range; wireless location systems

\section{INTRODUCTION}

Recently, diverse wireless location systems have been applied [1] [2]. The target and several measuring points are always on a plane in these wireless location systems. With the use of absolute-range measurement, the absolute distance between the target and measuring points can be calculated based on round-trip delay. Since the absoluterange measurement does not need to solve the synchronization problem, it has been widespread concern.

The measurement accuracy of ranging signal and the geometry between the target and several measuring points determine the positioning accuracy of the system [3] [4]. GDOP quantitatively describes the influence of the geometry on the positioning accuracy. Due to lower
GDOP often means higher measurement accuracy, all wireless location systems are searching lower GDOP.

It is shown that the lowest GDOP of $2 / \sqrt{N}$ can be achieved when the measuring points are located at the vertices of a regular polygon and the target at the center of the polygon in 2-D wireless location systems [4]. However, the kind of geometric relationship is idealistic, even under many practical conditions, such geometric relationship is not available.

Angle of coverage (AOC) is introduced to describe the geometry between the target and several measuring points in limited space. It is the central angle of the sector covering all the measuring points, where the target is placed at the center. When the AOC is less than $\pi / 2$, the lowest GDOP of $2 / \sqrt{N}$ is proved to be unreachable. And when $\mathrm{AOC}$ is equal to $\pi / 2$, a kind of geometry could achieve the lowest GDOP, where $\mathrm{N}$ is even. But when $\mathrm{N}$ is odd, the corresponding question has not been solved. In this paper, we not only propose a kind of geometry between the target and $\mathrm{N}$ measuring points to achieve the lowest GDOP, but also prove the necessary AOC realizing the lowest GDOP. On this foundation, the optimal geometry is derived as well as its new bound of GDOP, when the AOC is less than the necessary AOC.

The remainder of the paper is organized as follows. After a brief description of the GDOP and AOC in Section II, the necessary AOC to achieve the lowest GDOP is studied in section III. Furthermore, the optimal geometry of measuring points is derived when the AOC is less than the necessary AOC. The results of this paper are useful to design highly accurate wireless location systems, especially in the situation when the AOC is less than the necessary AOC.

\section{THE DESCRIPTION OF THE GDOP}

Consider an absolute-range-based 2-D wireless location system. It consists of a single stationary target 
located at $P_{t}=\left[x_{t}, y_{t}\right]^{T}$ and a set of $\mathrm{N}(N \geq 3)$ measuring points placed at locations $P_{i}=\left[x_{i}, y_{i}\right]^{T}, i=1,2, \ldots, N$. The $\mathrm{i}$-th measuring point is a distanced $d_{i}$ away from the target, where $d_{i}=\sqrt{\left(x_{i}-x_{t}\right)^{2}+\left(y_{i}-y_{t}\right)^{2}}$. Here the GDOP is as follow [4]:

$$
G D O P=\sqrt{\left[\left(H^{T} H\right)^{-1}\right]_{1,1}+\left[\left(H^{T} H\right)^{-1}\right]_{2,2}}
$$

Where $\left[\left(H^{T} H\right)^{-1}\right]_{i, i}$ denotes the $(\mathrm{i}, \mathrm{i})-$ th element of the matrix $\left(H^{T} H\right)^{-1}$

As shown in [4], there are two different expressions for the $\mathrm{H}$ in (1): one is for the absolute-range measurement, and the other for the pseudo-range measurement. Here the absolute-range measurement is considered. Therefore, the $\mathrm{H}$ in (1) is as follow [4]:

$$
H=\left[\begin{array}{cc}
\frac{x_{t}-x_{1}}{d_{1}} & \frac{y_{t}-y_{1}}{d_{1}} \\
\frac{x_{t}-x_{2}}{d_{1}} & \frac{y_{t}-y_{2}}{d_{1}} \\
\cdots & \cdots \\
\frac{x_{t}-x_{N}}{d_{1}} & \frac{y_{t}-y_{N}}{d_{1}}
\end{array}\right]=\left[\begin{array}{cc}
-\cos \alpha_{1} & -\sin \alpha_{1} \\
-\cos \alpha_{2} & -\sin \alpha_{2} \\
\ldots & \ldots \\
-\cos \alpha_{N} & -\sin \alpha_{N}
\end{array}\right]
$$

As shown in Fig.1, $\alpha_{1}(i=1,2, \ldots N)$ is the angle of the ray from the target to the $\mathrm{i}$-th measuring point relative to the positive $\mathrm{x}$-axis. Note that $\alpha_{j} \geq \alpha_{i}$ when $j>i$ $\forall i, j \in\{1,2, \ldots N\}$

Here the $\mathrm{AOC}$ is denoted by a variable $\beta$. Without loss of generality, let $\alpha_{1}=0$ and the signal emitted from the target covers a sector from 0 to $\beta$. Therefore, the $\beta$ and $\alpha_{i}(i=1,2, \ldots, N)$ satisfy the following relation:

$0=\alpha_{1} \leq \alpha_{2} \leq \ldots \leq \alpha_{N} \leq \beta$. The $\mathrm{AOC}$ and the geometry between the target and measuring points are shown in Fig.1.

The absolute-range measurement is usually implemented by a round-trip delay measurement. Recently, the absolute-range measurement has been widely used in range-based wireless location systems as it does not need consideration of the synchronization between the target and measuring points [5] [6].

Substituting (2) into (1), we have

$$
G D O P=\sqrt{4 N /\left[N^{2}-\left(\sum_{i=1}^{N} \cos 2 \gamma_{i}\right)^{2}-\left(\sum_{i=1}^{N} \sin 2 \gamma_{i}\right)^{2}\right.}
$$

\section{THE NECESSARY AOC AND THE OPTIMAL GEOMETRY}

\section{A. The Necessary AOC}

Proposition 1 . For any geometry between the target and the measuring points, the GDOP in (3) is low bounded by $2 / \sqrt{N}$, where $\mathrm{N}$ is the number of the measuring points [7].

Proof. For any $\alpha_{i}(i=1,2, \ldots N)$, it is held that

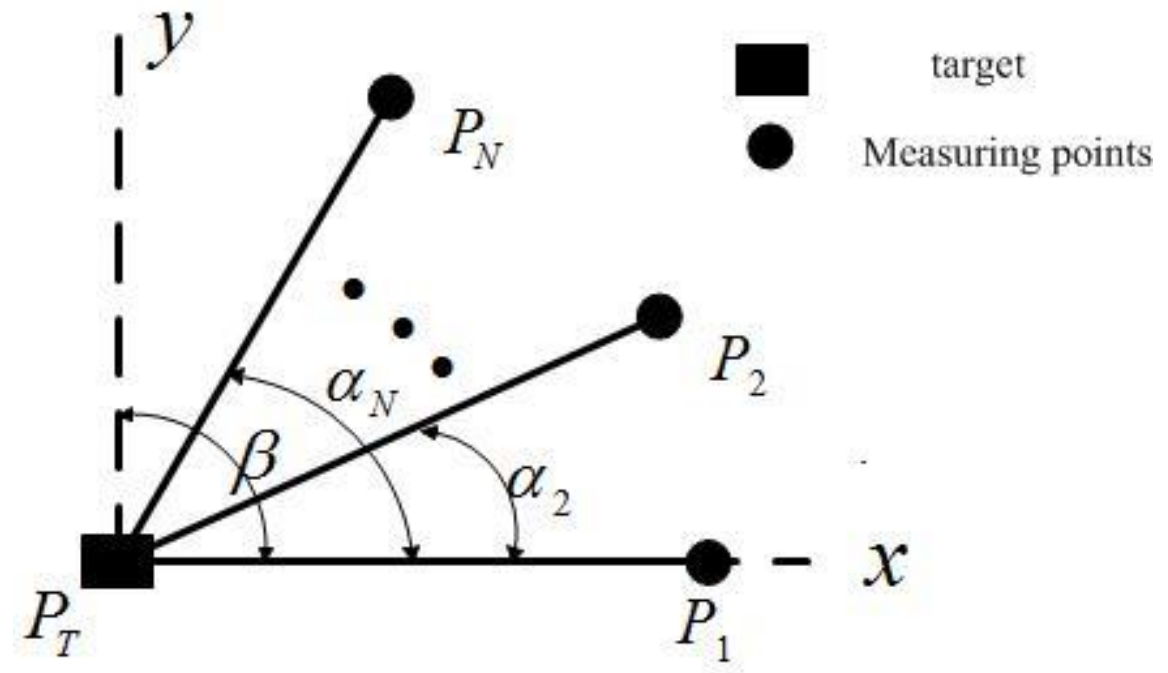

Figure 1. Angle of coverage of the geometry between the target and $\mathrm{N}$ measuring points. 


$$
\gamma=\left(\sum_{i=1}^{N} \cos 2 \alpha_{i}\right)^{2}+\left(\sum_{i=1}^{N} \sin 2 \alpha_{i}\right)^{2} \geq 0
$$

Using (3) and (4), we have $G D O P \geq 2 / \sqrt{N}$. The lowest GDOP of $2 / \sqrt{N}$ is achieved when the equality is achieved in (4). Note that the equality in (4) can be achieved, e.g., for

$$
\alpha_{i+1}-\alpha_{i}=2 \pi / N, i=1,2, \ldots N-1 \text {. }
$$

Proposition 2. The necessary AOC is $\pi / 2$ when $\mathrm{N}$ is even.

Proof: Let $\quad \alpha_{i}=0, \quad \alpha_{i+N / 2}=\pi / 2 \quad$, $i=1,2,3, \ldots, N / 2$.

Substituting the $\alpha_{i}, i=1,2,3, \ldots, N$ into (3), we find the GDOP achieve its minimum value $2 / \sqrt{N}$. We know the necessary AOC $\psi(N) \geq \pi / 2$ from proposition 2. So the necessary AOC is $\pi / 2$ when $\mathrm{N}$ is even.

Proposition 3. The necessary AOC is $\frac{\pi}{2}+\arcsin \frac{1}{N-1}$ when $\mathrm{N}$ is odd.

Proof: Based on the optimization theory, the geometry that $\frac{N-1}{2} \alpha_{1}, \alpha_{2}, \frac{N-1}{2} \alpha_{3}$ possesses the minimum AOC to achieve the lowest GDOP when $\mathrm{N}$ is odd. We put $\alpha_{1}=\alpha_{2}=\ldots=\alpha_{\frac{N-1}{2}}, \quad \alpha_{\frac{N+3}{2}}=\alpha_{\frac{N+5}{2}}=\ldots=\alpha_{N} \quad$ into equation (3), then we could get:

$$
\alpha_{N}-\alpha_{1}=\frac{\pi}{2}+\arcsin \left(\frac{1}{N-1}\right)
$$

Combined with proposition 2 and proposition 3, we can get the necessary AOC realizing the lowest GDOP:

$$
\psi(N)= \begin{cases}\pi / 2 & N=2 k \\ \pi / 2+\arcsin \left(\frac{1}{N-1}\right) & N=2 k+1\end{cases}
$$

B. The optimal sensor placement in limited observation space, where AOC is less than the necessary AOC We make

$$
G D O P=\sqrt{4 N /\left(N^{2}-\gamma\right)}
$$

$$
\text { Where } \gamma=\left(\sum_{i=1}^{N} \cos 2 \alpha_{i}\right)^{2}+\left(\sum_{i=1}^{N} \sin 2 \alpha_{i}\right)^{2}
$$

When $N$ is even

All the possible geometries between the target and measuring points can be described by $\alpha_{i}(i=1,2, \ldots N)$, which satisfy that $0=\alpha_{1} \leq \alpha_{2} \leq \ldots \leq \alpha_{N} \leq \beta$. Accord to the optimization theory, the optimal geometries at most have one angle that is not equal to the boundary. We assume the first $\mathrm{m}$ angles are equal to 0 and only the last $\mathrm{n}$ angles are equal to $\beta$. The special angle $\alpha_{i}\left(0 \leq \alpha_{i} \leq \beta\right)$, where $m+n+1=N$.Then we have

$$
\begin{aligned}
& \gamma=\left(m+\cos 2 \alpha_{i}+n \cos 2 \beta\right)^{2}+\left(\sin 2 \alpha_{i}+n \sin 2 \beta\right)^{2} \\
& >\left(\frac{N-1}{2}+\cos 2 \alpha_{i}+\frac{N-1}{2} \cos 2 \beta\right)^{2}+\left(\sin 2 \alpha_{i}+n \sin 2 \beta\right)^{2} \\
& =2(N-1) \cos \lambda \cos \left(\lambda-2 \alpha_{i}\right)+K
\end{aligned}
$$

Where $K=1+\frac{(N-1)^{2}}{2} \cos 2 \lambda$. It's easy to get: when $0 \leq \beta \leq \pi / 2$ and $m \geq n, \gamma\left(\alpha_{i}\right)$ is a monotone decreasing function, which means we can have a lower GDOP by enlarging $\alpha_{i}$ up to $\beta$ [8].

Similarly, $\gamma\left(\alpha_{i}\right)$ is a monotone increasing function when $m<n$, which means we can have a lower GDOP by reducing $\alpha_{i}$ down to 0 .

The results show that when the observation space is less than $\pi / 2$, the lowest GDOP will be achieved at

$$
\alpha_{i}= \begin{cases}0 & \mathrm{i}=1,2, \ldots,\lfloor N / 2\rfloor \\ \beta & \mathrm{i}=\lfloor N / 2\rfloor+1,\lfloor N / 2\rfloor+2, \ldots, N\end{cases}
$$

Where $\lfloor x\rfloor$ denotes the largest integer not greater than $x$.

The result show than all measuring points are place on both sides of the sector when the observation space is less than $\pi / 2$.

When $\mathrm{N}$ is even, the necessary observation is $\pi / 2$. According the above result, we have the optimal geometry and the corresponding GDOP, where the observation is limited.

$$
G D O P=\sqrt{8 /\left[N^{*}(1-\cos 2 \beta)\right]}
$$

When $N$ is odd 
Case 1, where $\beta<\pi / 2$

The necessary observation $\psi(N)>\pi / 2$. Here we get the optimal geometry from the equation (9), where the observation space $\beta<\pi / 2$, and the corresponding GDOP is:

$$
G D O P=\sqrt{8 N /\left[\left(N^{2}-1\right) *(1-\cos 2 \beta)\right]}
$$

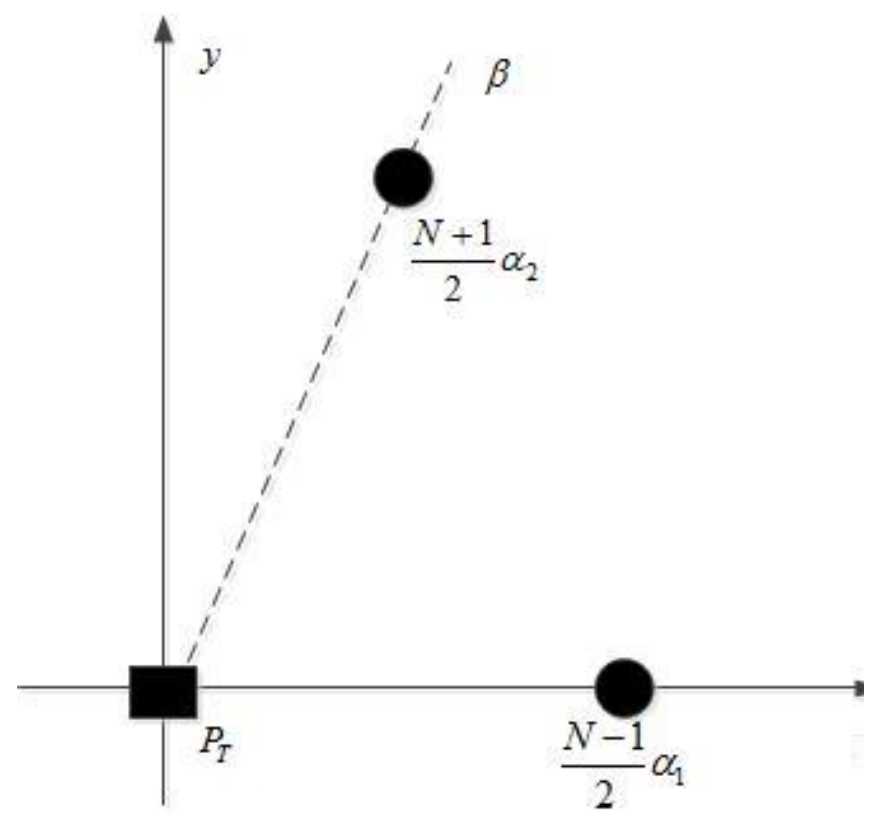

Figure 2. The optimal geometry in case 1.
Case 2 , where $\pi / 2<\beta<\psi(N)$,

$$
\begin{aligned}
& \gamma=\left(m+\cos 2 \alpha_{i}+n \cos 2 \beta\right)^{2}+\left(\sin 2 \alpha_{i}+n \sin 2 \beta\right)^{2} \\
& >\left(\frac{N-1}{2}+\cos 2 \alpha_{i}+\frac{N-1}{2} \cos 2 \beta\right)^{2}+\left(\sin 2 \alpha_{i}+n \sin 2 \beta\right)^{2} \\
& G D O P=2(N-1) \cos \beta \cos \left(\beta-2 \alpha_{i}\right)+K
\end{aligned}
$$

Where $K=1+\frac{(N-1)^{2}}{2} \cos 2 \beta$. Obviously, $\mathrm{K}$ is a constant.

It's obvious that the $\gamma$ achieve the maximum value when $\alpha_{i}=\beta / 2$. The GDOP is

$$
G D O P=\sqrt{4 N /\left[\left(N^{2}-1\right)(1-\cos 2 \beta / 2)-2(N-1) \cos \beta\right]}(
$$

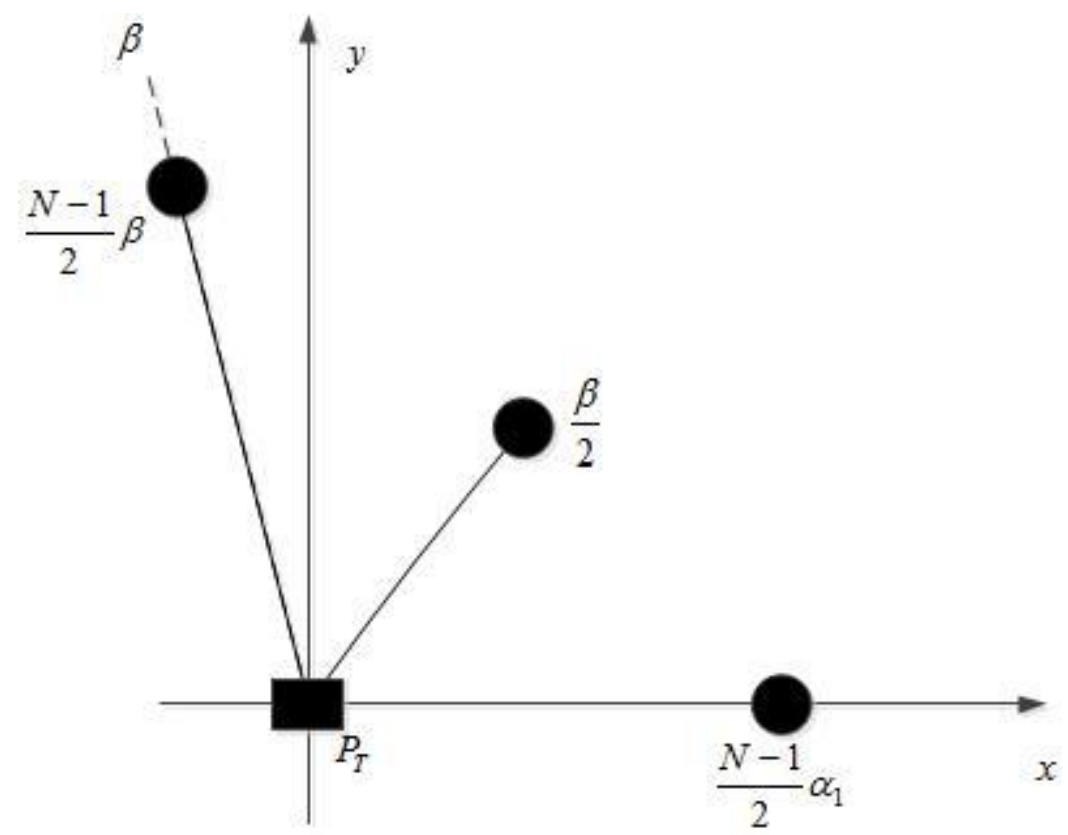

Figure 3. The optimal geometry in case 2 . 
Case 3, when $\beta=\pi / 2$,

The optimal geometry is not only one. Let $\alpha_{i}=0$, $\alpha_{i+(N+1) / 2}=\beta \quad, \quad i=1,2, \ldots(N-1) / 2, \quad$ and $\alpha_{(N+1) / 2} \in[0, \beta]$. Then we have the corresponding GDOP:

$$
G D O P=\sqrt{8 N /\left[\left(N^{2}-1\right) *(1-\cos 2 \beta)\right]}
$$

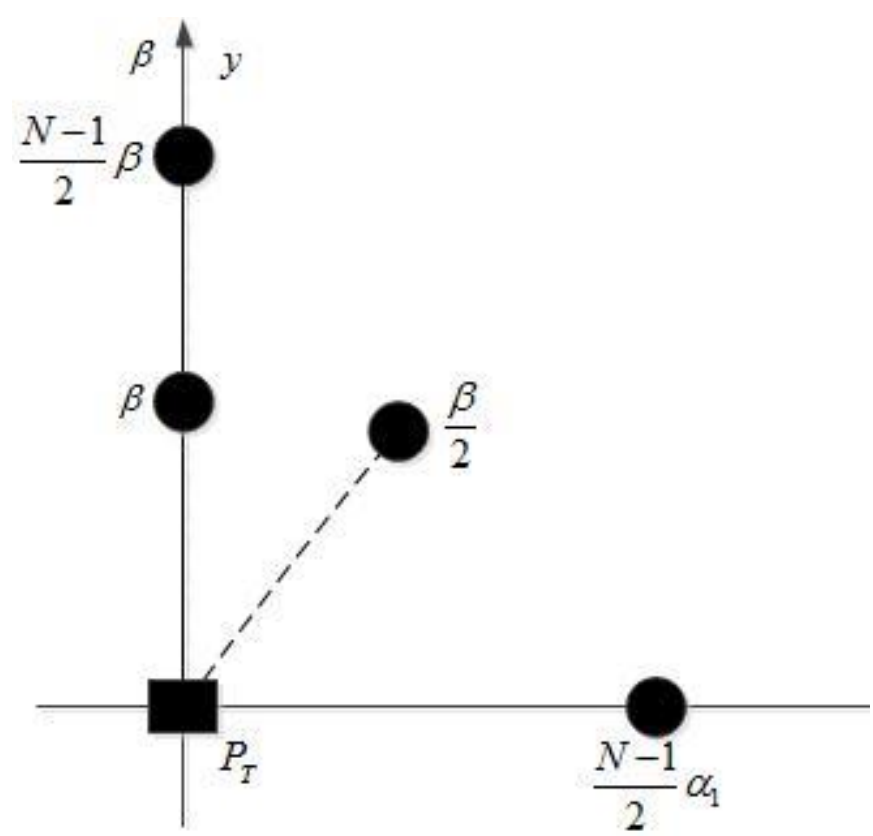

Figure 4. The optimal geometry in case 3 .

In case 3 , the low bound of GDOP is independent of the value of $\alpha_{(N+1) / 2}$. In fact, such characteristic realizes smooth transition between case 1 and case 2 .

\section{RESULTS}

The necessary AOC to achieve the lowest GDOP has been present. It is shown that the necessary AOC is the function of $\mathrm{N}$, where $\mathrm{N}$ is the number of the measuring points. The necessary AOC is equal to $\pi / 2$ when $\mathrm{N}$ is even, while the necessary AOC is monotonically decreasing to $\pi / 2$ when $N$ is odd. Obviously, $\pi / 2$ is the low bound of the necessary AOC. Then the optimal geometry of measuring points is derived as well as the new low bound of GDOP, when the AOC is less than the necessary AOC. The results of this paper are useful to design highly accurate wireless location systems, especially in the situation when the AOC is less than the necessary AOC, such as indoor positioning and special terrain positioning.

\section{ACKNOWLEDGMENTS}

This work is supported by the Wireless Signal Processing and Network Lab.

\section{REFERENCES}

[1] Marco Porretta, Paolo Nepa, Giuliano Manara, and Filippo Giannetti, "Location, Location, Location," IEEE Vehicle Tech. Magazine vol. 3, no.2, pp. 20-29, June 2008.

[2] Guoqiang Mao, Baris Fidan, Localization Algorithms and Strategies for Wireless Senor Networks, Information Science Reference, New York, 2009.

[3] X. W. Lv, K. H. Liu, and P.Hu, "Efficient Solution of Additional Base Stations in Time-of-arrival Positioning Systems," IET Electron. Lett. Vol. 46, no. 12, pp. 861-863, 2010.

[4] C. Levanon, "Lowest GDOP in 2-D Scenarios," IET Proc. Radar, Sonar Naving. Vol. 147, no. 3, pp. 149-155, June 2000.

[5] A. Bahillo, J. Prieto, S. Mazuelas, R. M. Lorenzo, J. Blas, and P. Fernandez, "IEEE 802.11 Distance Estimation Based on RTS/CTS Two-Frame Exchange Mechanism," Proc. IEEE Vehicular Tech Conference, Spring, 2009, pp.1-5.

[6] J.Wennervirta, and T. Wigren, "RTT Positioning Field Performance," IEEE Trans. On Vehicular Tech. vol. 59, no. 7, pp. 3656-3661, Sept. 2010.

[7] Qingyi, Quan, "observation space for lowest PDOP in absoluterange-based 2-D wireless location systems", IEEJ Transactions on Electrical and Electronic Engineering, 2014.

[8] S. Boyd, and L. Vandenberghe, Convex Optimization, Cambridge Univ. Press, 2004 